\title{
The Influence of Different Cross-Section Shapes of Train Body on Aerodynamic Performance
}

\author{
C.H. Liu, Dilong Guo, Shuanbao Yao, and Guowei Yang \\ National Key Laboratory of High-Temperature Gas Dynamics, \\ Institute of Mechanics of CAS, Beijing, China
}

\begin{abstract}
In this paper, the influence of different cross-section shapes of train body on aerodynamic performance has been investigated by numerical simulations. Computational fluid dynamics (CFD) is used with the second-order finite volume method with SST k-omega turbulence model. The results indicate that the aerodynamic performance is strongly affected by different cross-section shapes of train body, such as cross-sectional area, width-to-height ratio of crosssection, cross-section with straight or curved sides, etc.. Firstly, the drag coefficient and the overturning moment increase as the sides of cross-section become more curved. Secondly, the drag coefficient and the overturning moment are reduced as the width-to-height ratio of the cross-section is increased when the cross-sectional area is kept as a constant. Finally, air pressure pulse produced by two trains passing by in tunnel is approximately proportional to the cross-sectional area in a certain range and is independent to the cross-section shapes. Numerical results can be used for aerodynamic shape design of high speed trains.
\end{abstract}

Keywords: Air pressure pulse, cross-section shape, aerodynamic performance, numerical simulation.

\section{Introduction}

Along with the increasing velocity, the aerodynamic performance of a high speed train becomes more and more important. When streamlined train's speed reaches $250-300 \mathrm{~km} / \mathrm{h}, 75-80 \%$ of the total resistance is caused by external aerodynamic drag [1]. When a train runs under cross wind condition or two trains are passing by each other in a tunnel, due to the cross wind effect and air pressure pulse, the coefficients of aerodynamic drag force, lateral force, overturning moment and air pressure would make a significant change, which can greatly affect the comfort of passengers and the stability of trains. The aerodynamic performance is greatly influenced by the aerodynamic shape of a train [2-4]. At present, the streamlined shape of high speed trains has been studied intensively yet [5], however there are few references studying the influence of cross-section shapes of train body. In this paper, two scenarios are simulated based on six different cross-section shapes of train body. Firstly a detailed study has been performed on the relationship between aerodynamic drag coefficients, overturning moment coefficient and cross-section shape under cross wind condition. Secondly, considering the condition that two 
trains passing by each other, the relationship between air pressure pulse and crosssection shapes has been studied.

\section{The Geometry of Trains}

In order to study the aerodynamic performance of different cross-section shapes of train body, six shapes are discussed shown in Figure 1 and Figure 2. Table.1 shows details of each shape. To study the relationship between aerodynamic performance and cross-section with straight or curved sides and sectional area, four shapes are simulated. From Shape 1 to Shape 4, the value of $\alpha$ is taken bigger values gradually shown in Figure 3, which means the sides of section become more curved. Shape 5 can be obtained by rotating Shape 1 through 90 degrees, so Shape 5 shares the same sectional area with Shape 1. The effect of width and height on aerodynamic performance could be investigated through a comparison study between these two models. The sectional area of Shape 6 is the same as that of Shape 2, but Shape 6 has straight sides while Shape 2 has curved ones.
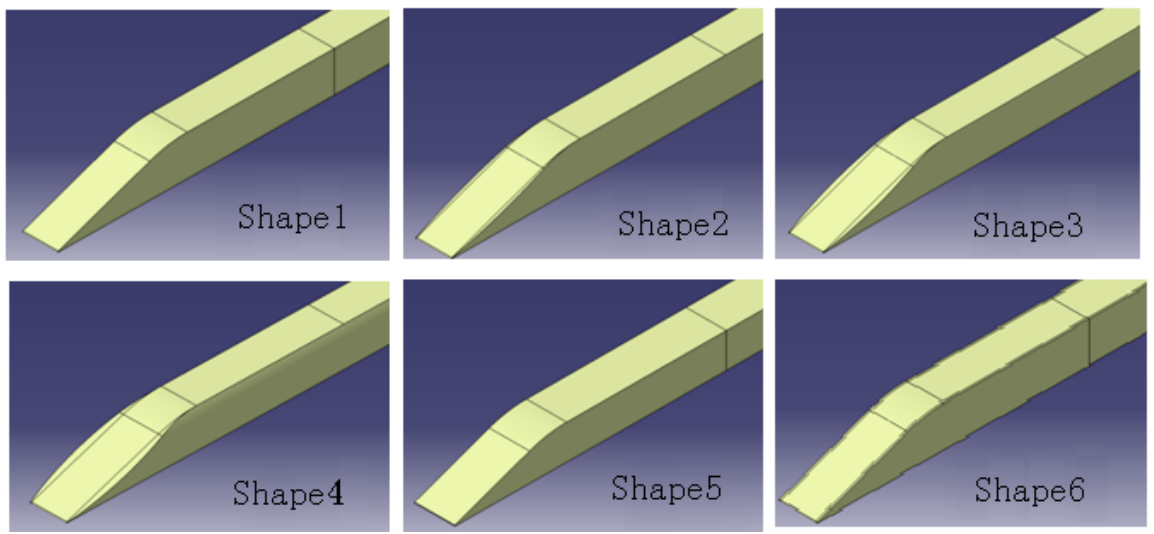

Fig. 1 Six shapes of train body

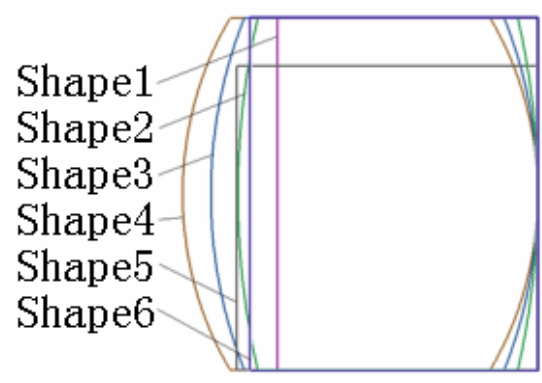

Fig. 2 Outlines of cross-section of train body

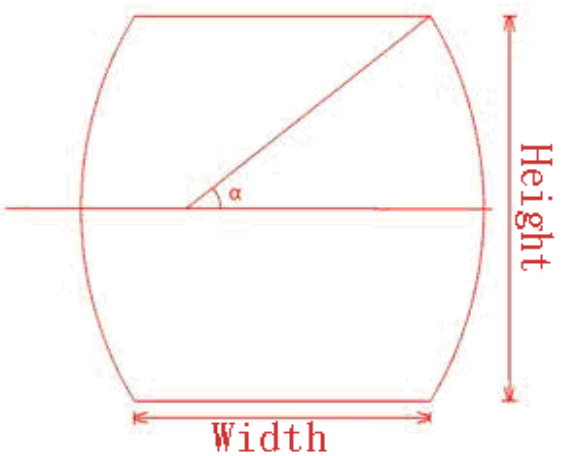

Fig. 3 The definition of $\alpha$ 
Table 1 Details of shapes of train body

\begin{tabular}{cccc}
\hline & Height $\times$ Width $(\mathrm{mm} \times \mathrm{mm})$ & Area $\left(\mathrm{m}^{2}\right)$ & $\alpha$ \\
Shape 1 & $3700 \times 3200$ & 11.84 & 0 \\
Shape 2 & $3700 \times 3200$ & 13.04 & 15 \\
Shape 3 & $3700 \times 3200$ & 13.88 & 25 \\
Shape 4 & $3700 \times 3200$ & 14.77 & 35 \\
Shape 5 & $3200 \times 3700$ & 11.84 & 0 \\
Shape 6 & $3700 \times 3200$ & 13.04 & 0 \\
\hline
\end{tabular}

\section{The Investigation on Cross Wind Effect}

The cross wind effect becomes crucial in combination with high running speed for a high speed train. In this paper, the finite volume approach has been adopted to simulate the flow field around the trains. From the results of the CFD simulations, cross wind effect is studied.

\subsection{Computational Domain and Grids}

All of the calculations are carried out for three car models with fore-, middle- and tail-bodies. The exterior boundary of computing zone is taken 5 times train length and 30 times cross-sectional height shown in Figure 4. The trimmer mesh is adopted. Figure 5 shows the minimum cell of train surface is $5 \mathrm{~mm}$. In order to accurately simulate the flow character in the boundary layers, 6-layers prism of $60 \mathrm{~mm}$ in height, 1.2 growth ratio is applied shown in Figure 6. In Figure 7 the systemic surface mesh is presented. The number of total grids is about 15 million.

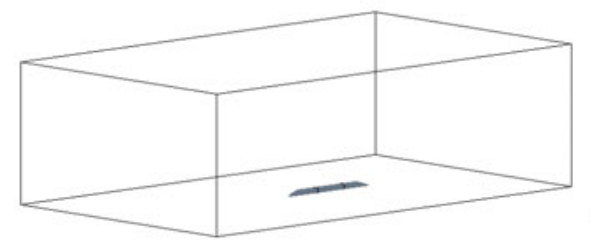

Fig. 4 Exterior boundary of computing zone

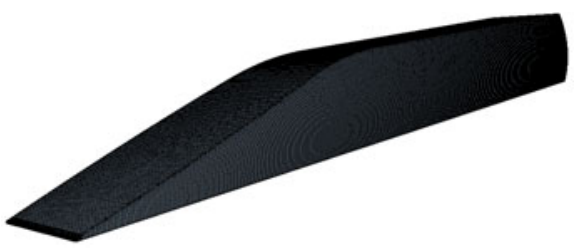

Fig. 5 Surface mesh of train-head 

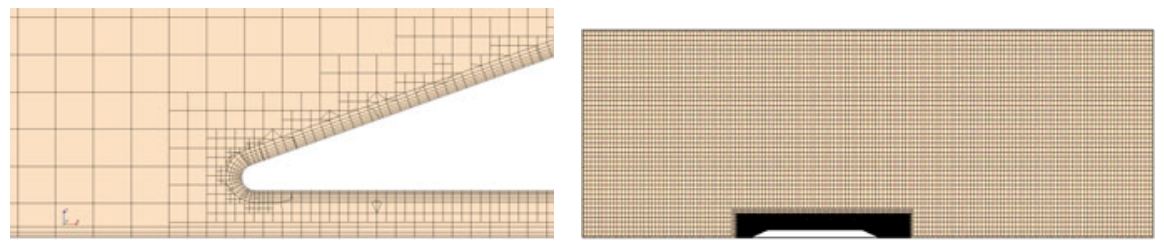

Fig. 6 Train-head mesh

Fig. 7 Systemic surface mesh of the whole domain

\subsection{Boundary Conditions}

In order to simulate a train with $350 \mathrm{~km} / \mathrm{h}$ under $15 \mathrm{~m} / \mathrm{s}$ cross-wind condition, the train speed is $98.37 \mathrm{~m} / \mathrm{s}$ and the yaw angle is 8.77 degree. No reflected far-field boundary condition is applied on the exterior boundary of computing domain. A relative moving wall boundary condition is adopted on the ground surface. On the surface of the trains, the no-slip wall boundary condition is applied.

\subsection{Computational Algorithms}

The CFD software Starcem+ is adopted in this paper. In order to correctly describe the flow field and obtain the wall-stress on the train surface, a SST k-omega turbulence model with wall functions treatment is adopted. The flow is considered as compressible flow and the second-order upwind method is used for the discretization of convective terms in Navier-stokes equations, while velocitypressure coupling has been performed with the Simple method.

\subsection{Results and Analysis}

Because of the cross wind effect, the flow field becomes extremely complex. The typical streamline at different cross sections for model 1 to model 5 is shown in Figure 8. There exist vortexes of various scales, however, two main separation zones locate on the train roof and leeward, respectively, thus, the flow under the train is sucked upwards by these separation vortexes. The vortex structures on the train leeward are similar as that of Karman vortex street on cylindrical flows.

For the model 1 to 4, as the curvatures of side surfaces become larger gradually, the vortex intensity is dramatically decreased. Because the height of the model 5 is smaller than other models, vortex intensity becomes smaller, which is benefit for the alleviation of the cross wind effect. 

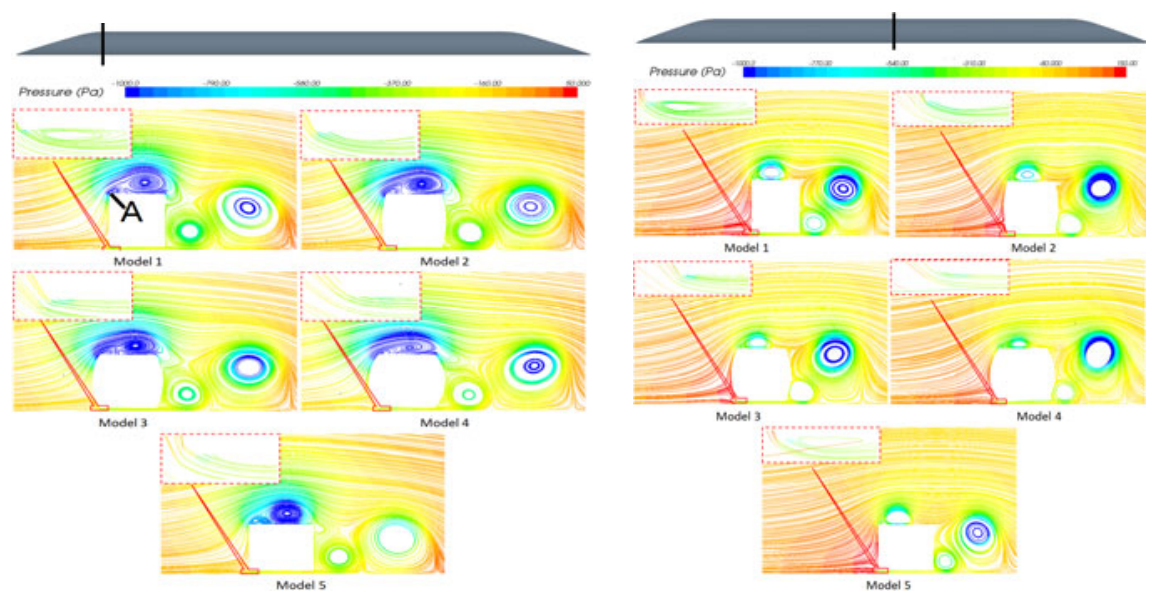

(a) 2D streamlines for cross section of train-head (b) 2D streamlines for cross section of train-body

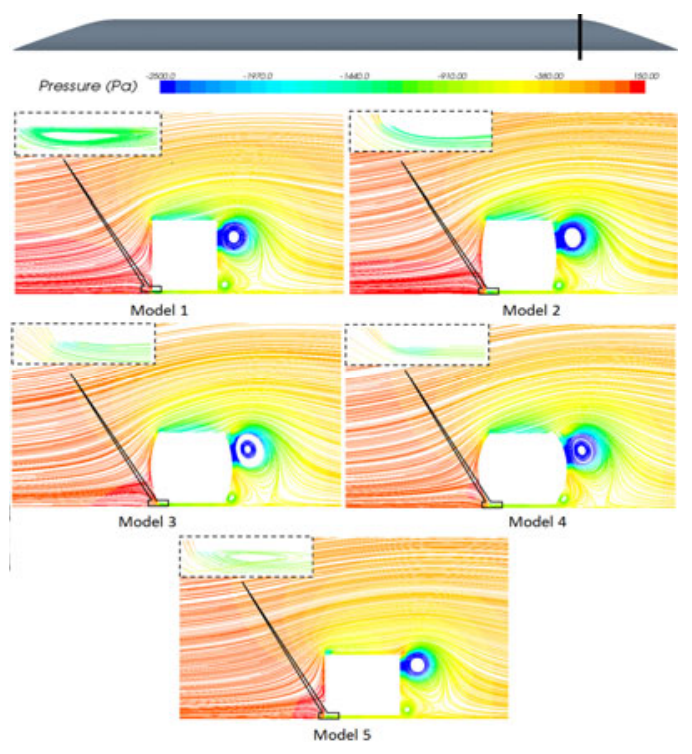

(c) 2D streamlines for cross section of train-tail

Fig. 8 2D streamlines for different cross sections

Figure 9 shows the pressure distributions of train surfaces of the five models. High pressure zone locates on the train-head surfaces. One low pressure zone locates at the joint part between train-nose and body due to flow speed-up, another low pressure zone on the leeward due to flow separation. 


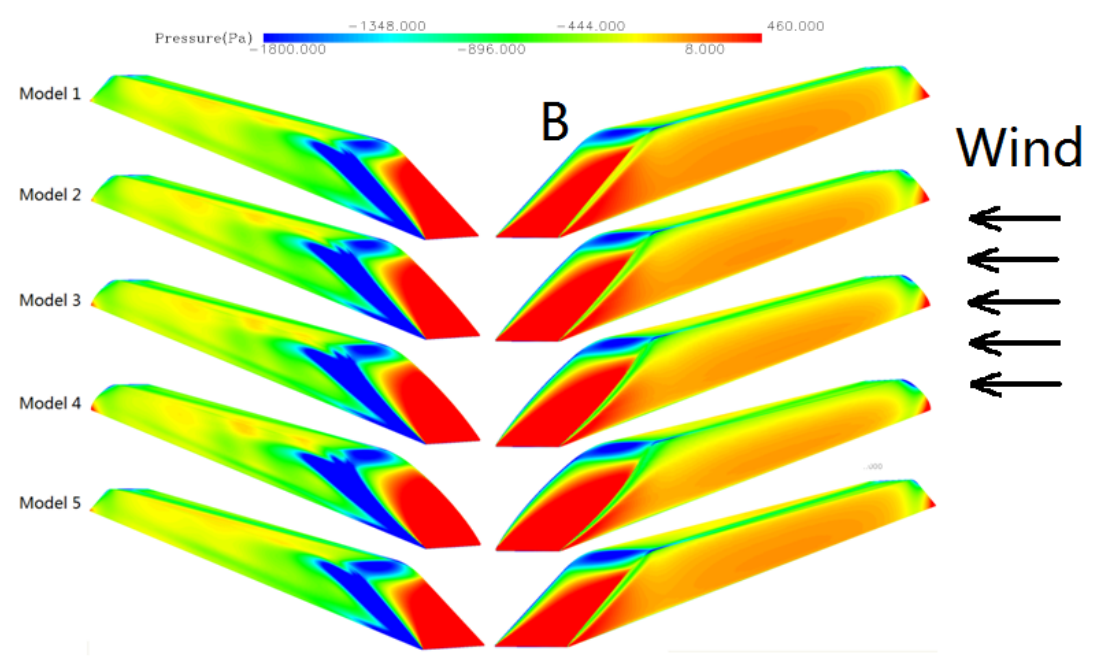

Fig. 9 Static pressure distributions of train surface

For the cross-wind case, the aerodynamic drag and overturning moment for the five models are presented in Figure 10 and Figure 11. The tail drag is larger than head. The total aerodynamic drag decreases as the train sides become more and more curved and Model 4 has the lowest drag. The total aerodynamic drag coefficient of Model 1 is the highest in all of the models and Model 5 has smaller drag than Model 1 due to lower train-height. The overturning moment coefficients of different models have the same trend. The total overturning moment coefficient of Model 1 is the highest of all models and Model 5 is lower than that of Model 1. Model 4 has the lowest overturning coefficient. The aerodynamic performance can be improved by the change of cross-section shape. In the Figure 8 has shown that curved sides of a train can make flow more fluently and generate weaker vortex in the leeward. Besides, when the width-to-height ratio of cross-section increases, the drag coefficient and the overturning moment decrease.

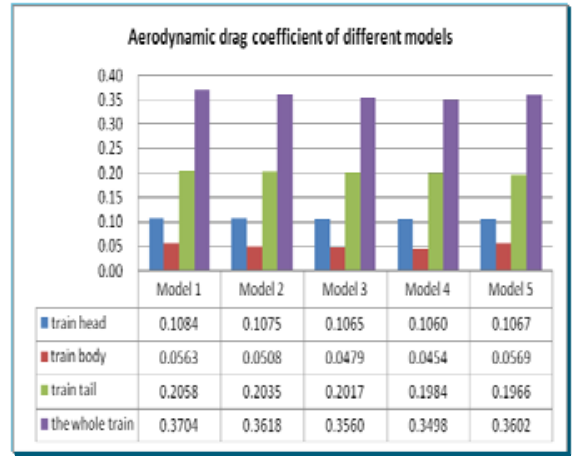

Fig. 10 Aerodynamic drag coefficient

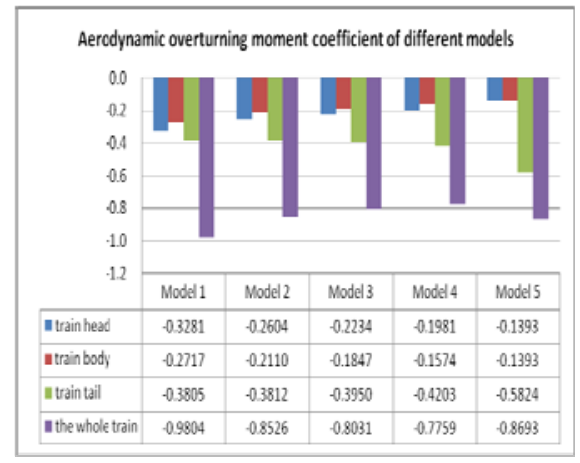

Fig. 11 Aerodynamic overturning moment coefficient 


\section{Air Pressure Pulse in Tunnel}

It is a common running environment that two trains pass by each other in tunnel. In here, CFD analyzes have also been adopted to simulate the flow field around the train and the pressure pulse on the train surface has been demonstrated.

\subsection{Computational Domain and Mesh}

The above six models with the length of $400 \mathrm{~m}$, which only increase the length of middle-body, are considered. The tunnel length is taken as the $2000 \mathrm{~m}$ with the cross-sectional area is $100 \mathrm{~m}^{2}$. The lateral distance between two trains is kept as a constant shown in Figure 12. Figure 13 shows that the exterior boundary at the entrances of the tunnel is taken as a semi-column with $600 \mathrm{~m}$ in length and $200 \mathrm{~m}$ in diameter. A hybrid mesh is adopted with a finer mesh closed to the train surface (the minimum cell dimension is $200 \mathrm{~mm}$ ) and a coarser mesh near to the exterior region (see Fig 14a). In Figure 14b, a view on the nose of the train can be seen. In order to better simulate the flow around the train, a prism mesh (the length is about $30 \mathrm{~mm}$ ) is adopted near the train region. For the purpose of simulating two trains passing by each other, the dynamic mesh technique has been utilized (see Figure 14c). The amount of total grids is about 400 million.

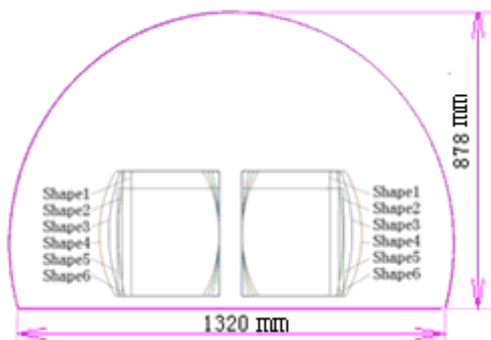

Fig. 12 Sectional shape of the tunnel

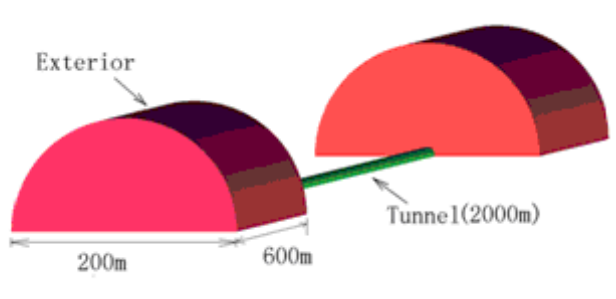

Fig. 13 Geometry of the tunnel and the exterior
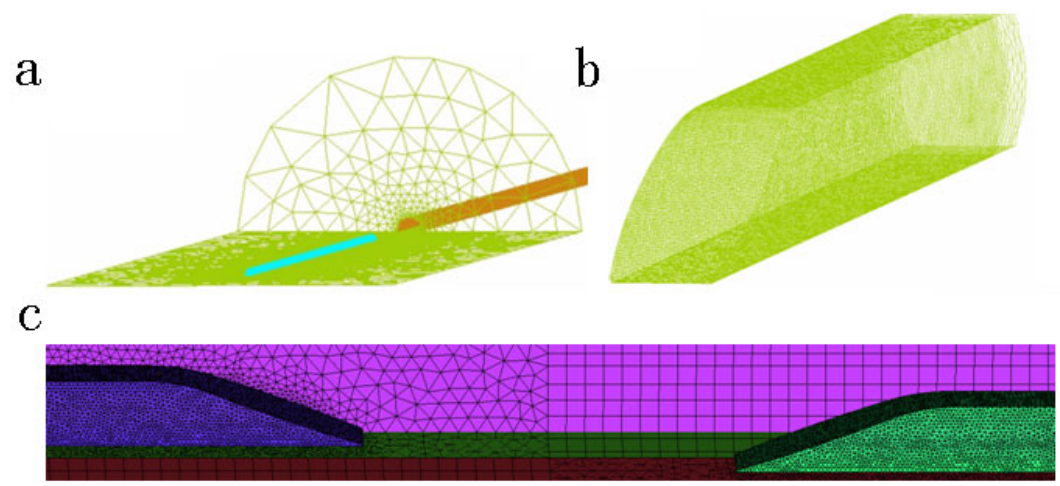

Fig. 14 Mesh of the tunnel and the train 


\subsection{Boundary Conditions and Monitor Points}

A pressure outlet boundary condition is applied on the exterior at the outer of the tunnel. The air has been set in standard condition: $\mathrm{P}=101325 \mathrm{~Pa}, \mathrm{~T}=288.15 \mathrm{~K}$, $\mu=1.7894 * 10-5 \mathrm{~kg} /(\mathrm{m} / \mathrm{s}), \rho=1.225 \mathrm{~kg} / \mathrm{m} 3$. A no-slip wall boundary condition is adopted on the tunnel surface and the ground which are both stationary. The surface grids of the trains are moving with the running speed of $350 \mathrm{~m} / \mathrm{h}$.

The air pressure pulse fluctuates violently when two trains pass each other in tunnel. Eight monitor points shown in Figure 15 have been distributed around the train (two monitor points on the train-head, three on the train body and others in the train-tail). Monitor points of $\mathrm{mh} 2$ and $\mathrm{mh} 3$ are on the joint part and the trainnose, respectively. The monitor mh1 locates in the interior side of the two trains.
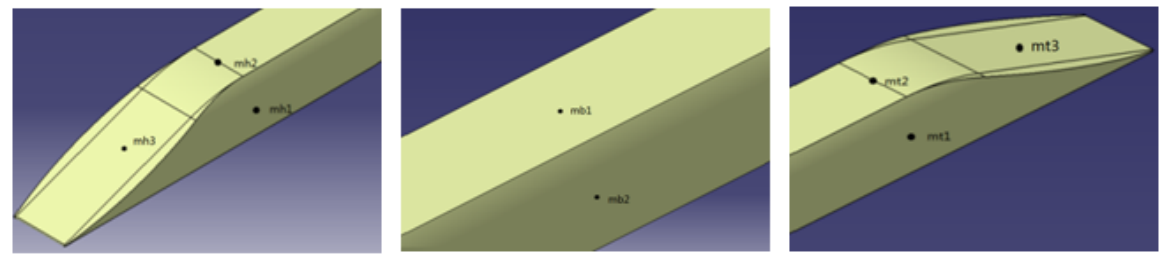

Fig. 15 Monitor points on the train surface

\subsection{Results and Analysis}

An instantaneous pressure contour just two train-heads passing by is shown in Figure 16. The high pressure zone occurs near the nose due to the stagnation of the air, and the lower pressure zone locates just after the nose due to the speed up of the flow. Figure 17 and Figure 18 are shown the instantaneous pressure distributions for the Model 1 to Model 4 entering into tunnel and just passing by in tunnel, respectively. In Figure 17 when trains are entering tunnel, initial compression wave is formed. The area of high pressure of Model 4 is the largest, indicating that Model 4 experiences the most serious air pressure pulse. In Figure 18 when trains are passing by in tunnel, the instantaneous pressure of the whole flow field is low. The absolute value of instantaneous pressure of Model 1 is the lowest which means Model 1 has the least influence on the tunnel.

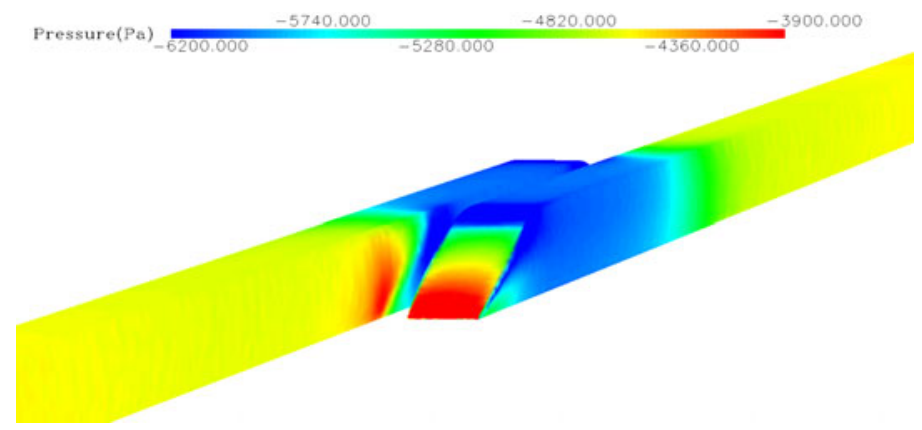

Fig. 16 Two trains passing by each other 

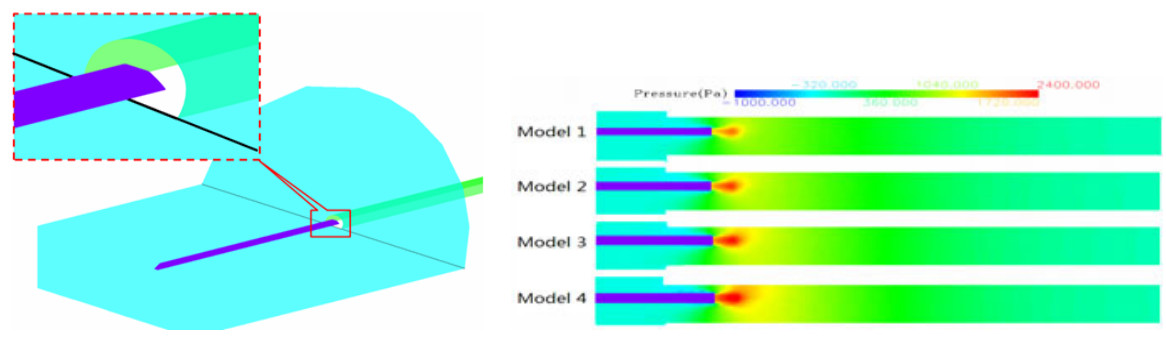

Fig. 17 Instantaneous pressure distribution around trains when trains enter the tunnel
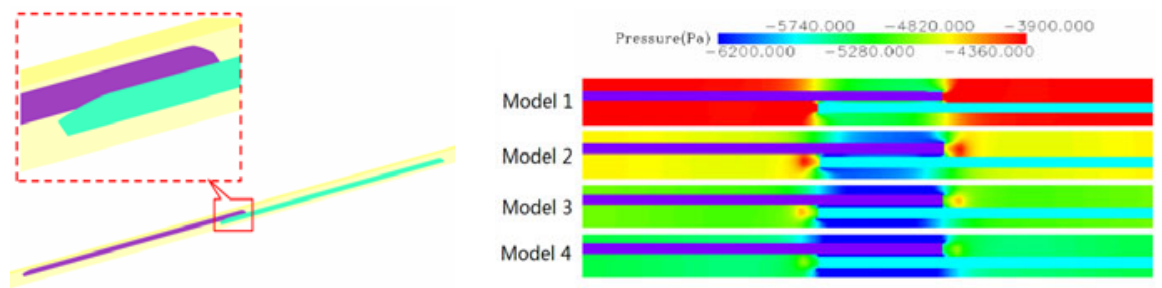

Fig. 18 Instantaneous pressure distribution around trains (The upper train is moving from left to right)

Figure 19 shows pressure history of different monitor points. The maximum pressure of train-head ascends at first then descends sharply, after that, it rises up quickly, finally goes down slowly. The minimum pressure has the same trend as the maximum one. This phenomenon can be explained. Firstly, the meeting train produces initial compression wave when it enters the tunnel which results in the first ascending stage. Secondly, the first descending stage is affected by three factors. (1) An expansion wave is reflected when the compression wave mentioned above reaches the entrance of the tunnel. (2) When the train-tail enters the tunnel, an expansion wave is generated. (3) The meeting train can also make an expansion wave. All these expansion waves join together, the air pressure reach the minimum which can be a limit pressure for trains design. Finally, the pressure changes followed are induced by the compression wave formed by expansion wave reflected and so on. For the train body and train-tail, the pressure shares some same characters with the train-head. However, the time when the monitor points on the train-tail enter the tunnel may be later than the time when the expression wave generated by the meeting train reaches the end, so the first ascending stage is not significant. 
a

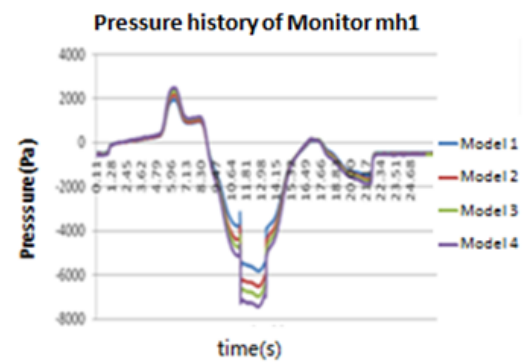

C

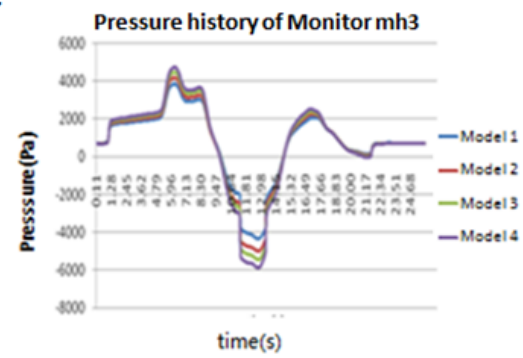

e

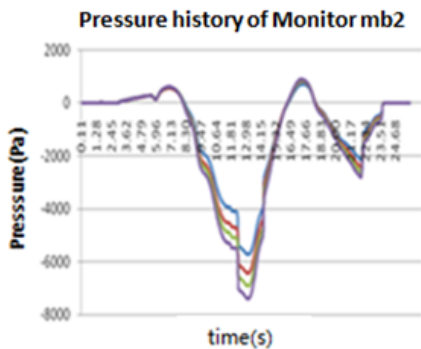

g

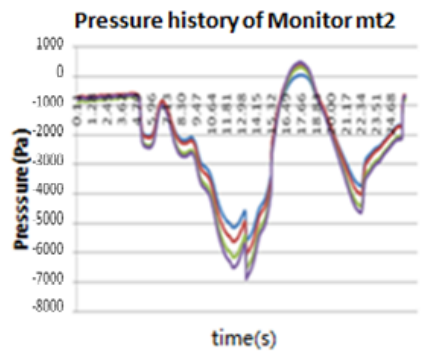

b

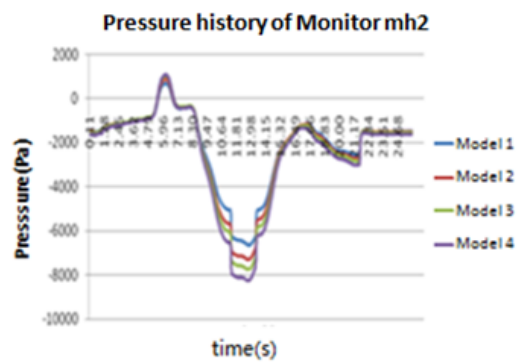

d

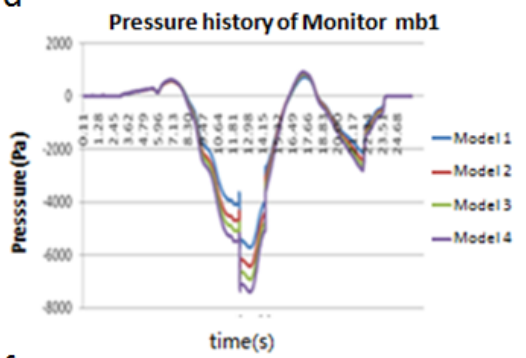

f

Pressure history of Monitor mt1

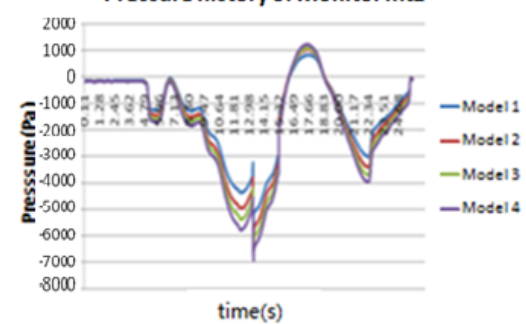

$\mathrm{h}$

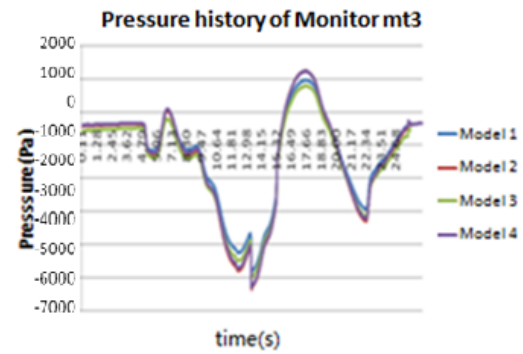

Fig. 19 Pressure history of monitor points

Fig 19 shows air pressure pulse can be affected by different cross-section shapes of train body. It is obvious that all the monitor points fluctuate violently. The maximum and minimum pressure of Monitor mh1 from Model 4 is $128 \%$ and $127 \%$ of those from Model 1 respectively. Figure 20 shows the maximum pressure 
ascends as the cross-sectional area ascends while the minimum pressure descends as the cross-sectional area descends. Figure 21 and Figure 22 shows Maximum and minimum pressure from some different models. The difference of pressure between Model 1 and Model 5 is insignificant. This means the value of width or height can affect little when the cross-sectional area is kept as a constant. Based on the results of Model 2 and Model 6, it can be deduced that it shares the same pressure distribution between the model with straight sides and the one with curved sides.

a

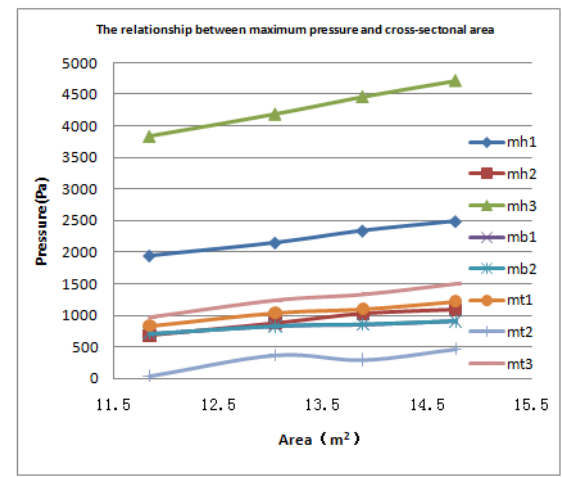

b

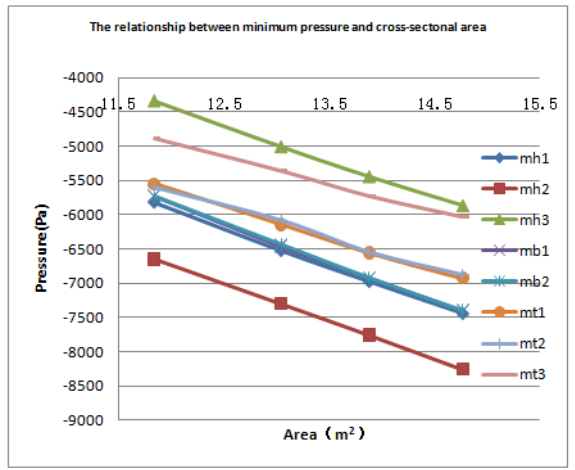

Fig. 20 Relationship between pressure and cross-sectional area
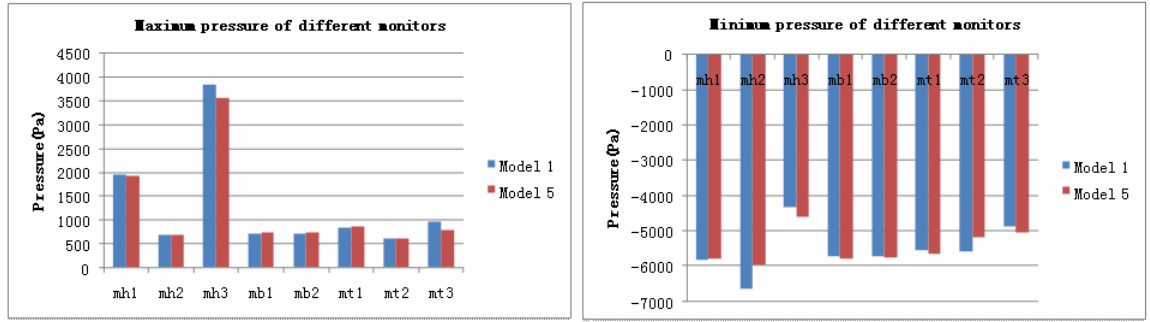

Fig. 21 Maximum and minimum pressure of Model 1 and Model 5
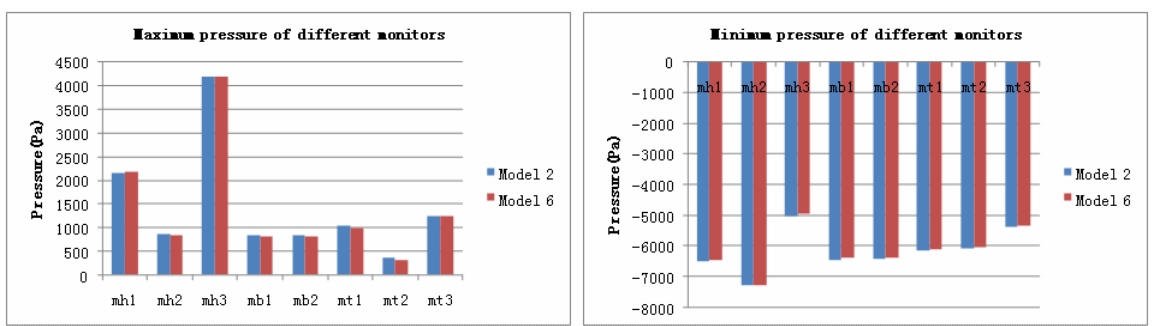

Fig. 22 Maximum and minimum pressure of Model 2 and Model 6 
The process of a train entering a tunnel can be compared to that of a piston entering a cylinder. When the blockage ratio becomes larger, the space between tunnel and train become smaller. So the air will be blocked more seriously and finally the air pressure pulse becomes more violently.

\section{Conclusions}

In this paper, the influences of different cross-section shapes of train body on the aerodynamic performance have been investigated. CFD results show that different cross-section shapes can greatly change the aerodynamic performance of a high speed train.

When trains run under the condition of cross wind, the aerodynamic drag and the overturning moment of a train with curved sides are lower than those of a train with straight sides. The drag coefficient and the overturning moment are reduced as the width-to-height ratio of the cross-section is increased when the crosssectional area is kept as a constant. When trains meet in a tunnel, air pressure pulse is approximately proportional to the cross-sectional area in a certain range and it independent to the cross-section shapes.

Numerical results can be used for aerodynamic shape design of high speed trains.

\section{References}

Joseph, A., Schetz, J.A.: Erodynamics of high-speed trains. Annual Review of Fluid Mechanics 33, 371-414 (2001)

Cheli, F., Ripamonti, F., Rocchi, D., Tomasini, G.: Aerodynamic behavior investigation of the new EMUV250 train to cross wind. Journal of Wind Engineering and Industrial Aerodynamics 98(4/5), 189-201 (2010)

$\mathrm{Xu}$, P., Tian, H.Q., Yao, S.G.: Integrative method of design and manufacture on streamlined head of train. Journal of Traffic and Transportation Engineering 1 (2007)

Diedrichs, B., Sima, M., Orellano, A., Tengstrand, H.: Crosswind stability of a high-speed train on a high embankment. Proc. Instn Mech. Engrs, Part F: J. Rail. and Rapid Transit. 221(F2), 205-225 (2007)

Kim, I., Ok, H.: A study on the aerodynamic characteristics of a high speed train entering a tunnel and frontal shape optimization. JKSAS 26(1), 17-26 (1998) 